\title{
The uninvited guests of our microbiome: Helicobacter pylori and Epstein-Barr virus and their role in gastric cancerogenesis
}

\author{
Magdalena Dzikowiec*1 ${ }^{\star}$ Dorota Pastuszak-Lewandoska²
}

${ }^{1}$ Department of Biomedicine and Genetics, Chair of Biology and Medical Microbiology, Medical University of Lodz, Poland

2 Department of Microbiology and Laboratory Medical Immunology, Chair of Biology and Medical Microbiology, Medical University of Lodz, Poland

Abstract

It is well established that human body is an ecosystem for numerous microorganisms: bacteria, fungi, eukaryotic parasites, and viruses. They form a "microbiome" that under conditions of homeostasis remains in a friendly mutual relationship with the host. However, the composition and diversity of this microbe community is dynamic and can be changed under the influence of environmental factors, such as diet, antibiotic therapy, lifestyle, and the host's genotype and immunity. The result of gut microbiome dysbiosis can lead even to cancer. The aim of this review is the description of the healthy gastrointestinal microbiome and the role of two infectious agents: Gram-negative bacteria Helicobacter pylori and Epstein-Barr virus in the development of gastric cancer in terms of gut dysbiosis.

H. pylori is the most important pathogen of gastric microbiome with clear impact on its diversity. Coinfection with Epstein-Barr virus causes chronic gastritis, and the inflammatory process is significantly increased. The process of carcinogenesis begins with chronic inflammation that causes atrophic gastritis, intestinal metaplasia, dysplasia, and finally cancer. It has been proven that chronic inflammatory infection caused by infectious agents increases the risk of stomach cancer. Molecular methods that are progressively used to explore the human microbiome provide hope that this knowledge will be used for future diagnoses and therapy in the state of its dysbiosis and in cases of gastric cancer.

Keywords

gastric microbiota $\bullet$ Helicobacter pylori $•$ EBV • gastric cancer $\bullet P P I$

Received: 13.08.2020, Accepted: 19.01.2021

\section{What is a microbiome, and what are the effects of gastric microbiome dysbiosis?}

Different parts of human body are inhabited by diverse microorganisms, which constitute a community described as microbiota, and the collection of genomes of distinct microbiota is called the microbiome. The microbiome is dominated by bacteria, but it also includes other microorganisms, such as archaea, fungi, viruses, and sometimes eukaryotic parasites.

The foundation for learning about the human microbiome was created by the Human Microbiome Project, implemented in 2007, aimed at sequencing the complete microbiome from 250 people and examining the interaction between microorganisms and the human body [1]. Although we are unable to culture most of the microbes inhabiting the human body, the development of molecular techniques has allowed us to better understand the human microbiome, primarily by sequencing $16 \mathrm{~S}$ mRNA.
The microbiome is extremely important from the point of view of metabolic processes, absorption of nutrients, and defense against pathogens. Microorganisms are not homogeneously distributed in the human body; in some places there are a lot of them, and in others they are present in very small amounts, depending on the local environment (biology of the area of the body (organ). Additionally, they can vary greatly at different sites within the same organ. The differences in composition as well as the density of microbiome correlates to an individual's health status, genotype, diet, and hygiene. Generally, the ratio of bacteria to human cells was thought previously to be $10: 1$ or even 100:1, but recently the ratio of bacteria to human cells has been updated to be closer to $1: 1$, which does not change the biological significance of the microbiome [2].

Within the gastrointestinal tract, there is a large quantitative and qualitative diversity of the microbial community, which is associated with the difference in the structure of the individual parts of the digestive tract. It is related to, among other things, oxygen conditions, $\mathrm{pH}$, and intestinal peristalsis. In the initial segment of the small intestine (duodenum and jejunum), few 
microorganisms can be found, due to the presence of bile and pancreatic juice, low $\mathrm{pH}$, and strong intestinal peristalsis. However, in the final segment of the small intestine, the ileum, the $\mathrm{pH}$ is higher (close to neutral), and it is inhabited by numerous, mostly relatively anaerobic bacteria. The large intestine, with slower peristalsis and $\mathrm{pH}$ closer to alkaline, has optimal conditions for the growth of most bacteria. Here is the most numerous group of microbes in the human body. In the stomach and small intestines a large percentage of microbiome is formed by facultatively aerobic microorganisms and in the large intestine by anaerobic taxa [3].

The stomach environment appears to be extremely unfavorable to most bacteria. Due to the low $\mathrm{pH}$ prevailing in the stomach, this organ was considered for a long time to be sterile. In 1982 the discovery of Helicobacter pylori from mucosa of patients suffering from peptic ulcer disease [4] changed the general opinion and motivated further search of other microorganisms inhabiting the stomach. The rapid flow of gastric contents in the upper gastrointestinal tract reduces the growth of microorganisms; in addition, secretory activity of the stomach and duodenum results in the extinction of most bacteria. It is assumed that there are about $10^{1}-10^{3} \mathrm{CFU} / \mathrm{g}$ in the stomach.

Despite an increasing amount of research on the human microbiome, most information concerns bacteria. The mycobiome has been shown to represent a large group of eukaryotic organisms. Fungi are a natural part of the microorganism community found in the human body, and although they make up a smaller proportion of the microbiota, their relationships with other microbes and the human body are not negligible. Most of the research work on the occurrence of fungi in the human body, their interaction with bacteria, and the effect of mycobiome dysbiosis on the occurrence of the disease state concerns the intestines $[5,6]$. Colonization of the human stomach by fungi can be favored by older age, malnutrition, H2-blocker therapy, the use of broad-spectrum antibiotics, and PPI [7]. The quantity of fungi in the human stomach is defined as $0-10^{2}$. Research using fungal DNA co-sequencing gastric juice analysis only allowed the identification of two genera of fungi Candida and Phialemonium [8].

Little research work is focused on the viruses that make up the microbiome. Retroviridae, often classified as human endogenous retroviruses (HERVs), predominate in the microbiome of the stomach. Herpesviridae are another group of viruses in high abundance, including Epstein-Barr virus (EBV), found in gastric mucosa. Also, bacteriophages of Staphylococcus, Burkholdera, and Enterobacteria were identified in the gastric microbiome [9].

Interactions between the microbiome and the human body are being increasingly studied, as well as the impact of changes in the composition of the microbiota on human health and disease. Studies on gnotobiotic animals have shown a very important role of microbiota in the organism's development and host physiology [10]. A kind of balance should be kept between microorganisms and the host, and any sudden disturbance in the composition of the microbiota-a condition called dysbiosis-can be associated with the occurrence of disease symptoms, sometimes even with the development of cancerous lesions. The process of carcinogenesis may be affected by the disturbance of signals stimulating and inhibiting the development of inflammation, the cytotoxic effect, excessive proliferation of epithelial cells, and the production by microorganisms of toxic, intermediate products of metabolism that can cause damage to epithelial cells [10]. The change in the structure of the gastrointestinal microbiome may be the result of many factors, including diet, stress, medicine (mainly antibiotics), and smoking.

Changes in the gastric mucosa, such as ulcers, promote the growth of Candida albicans. Gastric ulcers associated with the colonization of Candida albicans are well documented in the literature [11]. However, it is difficult to say whether Candida is an etiological factor causing ulcers or whether its presence in the stomach is a secondary effect. Also, the cooccurrence of $H$. pylori and $C$. albicans may act synergistically in the development of peptic ulcers [7, 8]. Karczewska et al. [12] showed that $66.7 \%$ of patients with gastric disorders with Candida in the mucosa were also colonized by $H$. pylori. There have also been studies confirming the presence of $H$. pylori inside Candida cells, its ability to survive in Candida vacuoles and the possibility of expressing $H$. pylori-specific proteins, e.g., VacA, urease or peroxiredoxin. The relationship between $H$. pylori and Candida provides bacteria protection against adverse gastric conditions, including low $\mathrm{pH}$, the presence of antibiotics, and also protects with nutrients [8, 13]. It has been also shown that growth of Lactobacillus can counteract colonization of the stomach by Candida by producing hydrogen peroxide and organic acids.

The etiopathogenesis of many cancers is still not well understood. It is estimated that about $15 \%-20 \%$ of all cancers can be associated with the presence of an infectious agent. Infectious agents affect cell growth, destabilize the host's immune system, and lead to changes in cells resulting in long-term infection [14]. Increasing attention is being paid to the association of inflammation with the change in the composition of the microbiota and the development of cancer [15]. Infections cause chronic inflammation, which can lead to carcinogenesis $[16,17]$. So the tumor formation process may be caused by the presence of an infection caused by some viruses, bacteria, and even parasites.

Gastric cancer is one of the most common cancers in the world with the highest mortality rate. Most often, it is diagnosed at an advanced stage, which results in poor prognosis [18, 19]. Gastric cancer is fifth among the most frequently diagnosed 
cancers and third in terms of mortality in the world [20]. Cases of stomach cancer are most commonly reported in East Asian countries, including Korea, Japan, and China, where about half of all new cases are diagnosed, over four times higher than in Western Europe; in Korea, for example, gastric cancer cases have a frequency of 33.8 per 100,000 [21]. This type of cancer is characterized by a relatively low five-year relative survival-about 32\% (2010-2016) [22].

Gastric cancer can be divided into subtypes by specific epidemiology and risk factors and recently also by molecular profiles. The Cancer Genome Atlas (TCGA) [23] distinguished four subtypes of gastric cancer taking into account the underlying mechanisms: Epstein-Barr virus (EBV)-positive, tumors with microsatellite instability, and that which is genomically stable but has chromosomal instability. Noteworthy is the separate subtype of gastric cancer associated with EBV infection, which means the importance of external pathogens in the disease.

\section{Normal gastric microbiota}

The microbiota of the stomach and duodenum shows more dynamic composition than the intestinal microbiota. From the microbiota studies of healthy people, it was found that gastric microbiota shows high interindividual diversity as compared with the duodenum or any segment of the intestine that shows the least dissimilarity [24].

Five phyla have been identified in the stomach: Firmicutes, Bacteroidetes, Actinobacteria, Fusobacteria, and Proteobacteria, while Prevotella, Streptococcus, Veilonella, Rothia, and Haemophilus are the most common at the genera level [25]. There is no clear answer as to whether the stomach is uniformly inhabited by the same bacteria. According to some authors, there is no difference in microbiota between the antrum and the corpus, only in case of gastritis a decrease in Prevotella is observed in the antrum; while other authors distinguish the dominance of Proteobacteria, Firmicutes, and Bacteroidetes in the antrum and Firmicutes, Proteobacteria, Fusobacteria, and Bacteroidetes in the corpus [26].

Many bacteria have been detected in gastric fluid, such as Streptococcus, Neisseria, and Lactobacillus, but it is difficult to determine whether they belong to transient microbiome or colonize the stomach. Microorganisms travel through the gastrointestinal tract from the mouth; as a result, more and more research is focusing on the gastric mucosa [27]. Studies using methods from molecular biology have confirmed the presence of previously isolated bacteria by classical methods (Lactobacillus, Streptococcus, Veilonella, Escherichia) but have also discovered the presence of bacteria not previously included in the microbiota of the stomach: Prevotella,
Caulobacter, Actinobacillus, Corynebacterium, Rothia, Gemella, Leptotrichia, and Deinococcus [28].

The effect of $H$. pylori on the composition of the stomach microbiota has been followed in many studies. In the initial period of colonization by $H$. pylori, in response to inflammation, the secretion of hydrochloric acid increases as a result of stimulation of gastrin secreting $G$ cells. However, in the chronic phase, degenerative changes of the epithelium and glands of the mucosa occur, which secondarily results in mucosal atrophy and acidosis; therefore, this process leads to an increase in $\mathrm{pH}$, facilitating the colonization of other microorganisms [14, 29, 30]. After colonization of the stomach by $H$. pylori, it becomes the dominant species in the stomach microbiota [31].

According to some researchers, there is a higher bacterial diversity in $\mathrm{H}$. pylori-negative patients, while others report no changes related to the status of $H$. pylori. Brawner et al. [32] observed differences in the change in microbiota related to the status of $H$. pylori only in children: in those who were $H$. pylori-positive there was a greater variety and abundance of non-Helicobacter, Proteobacteria, and there was a lower quantity of Firmicutes than in adult patients. The results of other studies confirm the differences in the composition of the microbiota between $H$. pylori-negative and $H$. pylori-positive persons. In $\mathrm{H}$. pylori-negative patients Firmicutes dominates, followed by Actinobacteria, Bacteroidetes, Proteobacteria, Fusobacteria in last place, and in $\mathrm{H}$. pylori-positive patients Bacteroidetes significantly dominates, whereas the others are in low abundance. It follows that $H$. pylori-negative people had more complex and highly diverse microbiota [33].

A relationship has been also demonstrated between the abundance of $H$. pylori in the stomach and intestinal microbiota. The abundance of $H$. pylori was associated with a lower amount of Bifidobacteria and a higher amount of Candida albicans in the intestine, as well as with the degree of dysbiosis in the intestine [34].

The discovery of Helicobacter pylori and other stomach colonizing microorganisms has highlighted the importance of microbiome in maintaining healthy body balance and disease development when the biodiversity and the quantity of microorganisms are disturbed.

\section{How the microbiota changes in gastric cancer}

Patients' stomach microbiota has been shown to change during progression to gastric cancer. Eun et al. [35] observed a change in the species abundance of gastric microbiota in gastric cancer compared with the microbiota of intestinal metaplasia patients and those with chronic inflammation. During the development of cancer, the diversity of the stomach microbiota decreases. A significant decrease in 
the Epsilonproteobacteria class and the Helicobacteriaceae family and an increase in the Bacilli and Streptococcaceae classes were observed in patients with gastric cancer. Similar changes were also reported by Aviles-Jiminez et al. [36], who found a decrease in bacterial richness along with non-atrophic changes from intestinal metaplasia to gastric cancer. Hu et al. [37] observed a significant increase in Betaproteobacteria and Gammaproteobcteria and a decrease in Alphaproteobacteria in gastric cancer compared with superficial gastritis. Noteworthy is the significant increase in abundance of Sphingobium yanoikuyae in superficial gastritis, which has the ability to break down xenobiotic compounds, especially aromatic hydrocarbons that have toxic, genotoxic, mutagenic, and carcinogenic effects. Negative association with advanced gastric adenocarcinoma has been demonstrated [37]. Research confirms that Helicobacter pylori is predominant in the initial stages of the cascade of changes in the mucosa, and in later stages of the intestinal metaplasia, dysplasia, and adenocarcinoma the abundance of Helicobacter decreases, but new microorganisms then appear-Proteobacteria (including Escherichia, Shigella, and Burkholderia), Firmicutes (Lactobacillus, Lachnospiraceae, Streptococcus and Veilonella), and Bacteroidetes (Prevotella) $[25,26,33]$. It was confirmed by the research of Ferreira et al. [38], who examined the microbiota of 135 people-54 with gastric carcinoma and 81 with chronic gastritis (nextgeneration sequencing of the 16S rRNA was used). In patients with chronic gastritis, Helicobacter had the highest abundance, and Streptococcus, Prevotella, and Neisseria also appeared in large quantity. In contrast, gastric carcinoma showed a decrease in Helicobacter abundance, while several taxa were definitely more abundant-Citrobacter, Clostridium, Lactobacillus, Achromobacter, Rhodococcus, and Phyllobacterium.

In the research of Castano-Rodrigues et al. [39], the composition of the gastric microbiota was compared between people with functional dyspepsia and gastric cancer: 91 taxes were different, and the presence of $H$. pylori had an impact on the composition of the microbiota. A significant difference was found for 38 operational taxonomic units (OTU), 23 of them were enriched in gastric cancer, including Lactococcus OTU0002, Fusobacterium OTU0087, Pasteurellance unclassified OTU0022, Staphylococcus OTU0045, Methylobacterium OTU0018, Veillonella OTU0005, Dialister OTU0132, and Leptotrichia OTU0042.

Interesting results were obtained from the gastric microbiota study in Colombia, in which two populationswith low (Tumaco) and high cancer risk (Tuquerres) were considered. In the Tuquerres population, a significant amount of Leptotrichia wadei and Veillonella sp. was noticed, whereas
Tumaco was more abundant with bacteria recognized as a normal element of human microbiota, including Staphylococcus sp. [40]. Studies in the Korean population involving 556 people (268 patients with gastric cancer and 288 controls) showed that people who had high levels of Helicobacter pylori and Cutibacterium acnes were more likely to develop stomach cancer. In addition, the presence of Prevotella capri in patients increased the risk of cancer as compared with noncarriers. Research results point to $H$. pylori, Prevotella capri, and Cutibacterium acnes as strong risk factors and also suggest that Lactococcus lactis may be a protective factor in the development of stomach cancer, as its abundance was significantly higher in control than in cancer patients [21]. Interestingly, in the research of Hsieh et al. [41], attention was paid to the genera of Clostridium, Fusobacterium, and Lactobacillus, regardless of their role in the oncogenesis process, stressing, however, that their overabundance could be a diagnostic tool in gastric cancer.

Additionally, bacteria usually colonizing the oral cavity as commensals or opportunistic pathogens, including genera Neisseria, Alloprevotella, and Aggregatibacter, species Streptococcus mitis, S. oralis, S. pneumoniae, and the Porphyromonas endodontalis strain, were also found in gastric cancer [37]. It has been reported that oral bacteria were more abundant in gastric cancer compared with intestinal metaplasia and superficial gastritis. Attention was paid to several species of oral microbiota-Streptococcus anginosus, Peptostreptococcus stomatis, Parvimonas micra, Slackia exigua, and Dialister pneumosintes-they may play a key role in the development of the carcinogenesis process and could be used as noninvasive biomarkers to distinguish gastric cancer from superficial gastritis [42]. Other authors also note that the high level of periodontal pathogen colonization is associated with an increased risk of gastric precancerous lesions $[43,44]$. A very interesting study was conducted by Sun et al. [45], which aimed to examine the characteristics of the oral microbiome in patients with gastric cancer and introduced screening tests for stomach cancer by using oral microbiome. It has been shown that changes in oral microbiome can be used as an indicator for gastric cancer in diagnostics. The following taxonomic units were considered in the assessment panel: Veillonella, Prevotella, Leptotrichia, Rothia, Capnocytophaga, Aggregatibacter, Campylobacter, Megasphaera, Tannarella, and Granulicatella. Moreover, the research by Wu et al. [46] indicated that even the microbiome of the tongue could be used in the early detection and prevention of stomach cancer. It was found that the greater abundance of Firmicutes and lower abundance of Bacteroidetes were associated with an increased risk of gastric cancer. 


\section{Helicobacter pylori and its association with gastric cancer}

A huge part of the research focuses on the participation of individual pathogens such as Helicobacter pylori and its ability to initiate disease such as gastritis or gastric cancer. Almost half of the world's population is made up of carriers of $H$. pylori. In recent decades, a decrease in prevalence has been observed, which may be related to the improvement of socioeconomic conditions [47]. However, in developing countries, $H$. pylori infection is still a big problem that affects about $80 \%$ of the people [31].

Initially, it was called Campylobacter pylori, while after the discovery of morphological, biochemical, and genetic differences between Campylobacter pylori and other bacteria of this genus, a new genus Helicobacter was created in 1898, and the human-isolated bacterium was called Helicobacter pylori. H. pylori belongs to the $\varepsilon$-proteobacteria and is Gramnegative, spiral shaped, microaerophilic flagellatum bacteria. Despite the relatively unfavorable conditions in the stomach, this is the only known $H$. pylori habitat. The bacterium has unique adaptations for survival and proliferation in the stomach environment, including urease activity, ability to penetrate through the gastric mucus layer (spiral shape, presence of flagella, mucolytic enzymes, the production of host mucin), and binding of bacterial surface adhesives to the corresponding receptors of the gastric epithelial cells [30, 31]. All clinical pathogenic strains are urease-positive, while urease-negative strains that are nonpathogenic can be found only in laboratory culture [29].

In 1994, the International Agency for Research on Cancer (IARC) recognized Helicobacter pylori as a class I carcinogen. $H$. pylori-induced carcinogenesis is primarily due to the expression and activity of VacA vacuolizing cytotoxin that is responsible for vacuolization of eukaryotic cells, and the activity of the CagA protein encoded by cag PAI pathogenicity island. The tumorigenic process is associated with the polymorphism of PAI genes. CagA interacts with host proteins via a dependent and independent phosphorylation pathway causing changes in motility, cell proliferation and polarity, resulting in a phenotype change of eukaryotic cells $[14,48]$. The PAI also includes genes whose products build the so-called type IV secretion system (T4SS) responsible for the direct transfer of CagA toxin to the cytoplasm of gastric mucosal epithelial cells [49]. Additionally, VacA and CagA produced by $H$. pylori were detected in hepatic tissue with hepatocellular carcinoma, and it was additionally found that bacterial LPS promotes the growth and migration of liver cancer by increasing the level of IL-8 and TGF- $\beta 1$ [50].

Colonization of the gastric mucosa by $H$. pylori initially involves the antral part, and over time it spreads to the entire mucosa initiating the inflammatory process. Each $H$. pylori infection ultimately leads to the development of chronic gastritis; however, most cases are without a complaint and do not require treatment. Only about $15 \%$ cases have symptoms [31].

Also, chronic inflammatory infection caused by $H$. pylori increases the risk of stomach cancer more than threefold [51]. Although, $1 \%-2 \%$ of people infected with $H$. pylori develop stomach cancer. The process of carcinogenesis begins with chronic inflammation that causes atrophic gastritis, intestinal metaplasia, dysplasia, and finally cancer. $H$. pylori usually colonizes the gastric antrum and causes antrum-predominant gastritis in most cases. The development of gastric mucosal lesions is known as Correa's gastric cancerogenesis cascade $[26,52]$.

\section{Epstein-Barr virus (EBV) and its association with gastric cancer}

Viruses such as EBV, CMV, and HHV6 were detected in gastric tumors, but among them EBV is the most important. EBV is one of the most common viruses in the world, and around $90 \%$ of the world's population has anti-EBV antibodies. Infection is usually asymptomatic [53]. The pathogen has the ability to determine latency in B lymphocytes. A decrease in host immunity may cause reactivation of the infection and make the virus enter the lytic cycle. EBV belongs to oncogenic viruses that promote proliferative processes. Burkitt's lymphoma and squamous cell carcinoma of the nasopharynx are among the most frequently mentioned EBV-related diseases, but a relationship with the development of gastric cancer and Hodgkin's lymphoma has also been shown [54, 55, 56].

EBV infection in the development of stomach cancer is associated with abnormal methylation of tumor suppressor genes, such as RUNX1, RBM5, and PSME1 [15]. Virus proteins involved in the process of carcinogenesis, such as EBNA-1, EBNA-2, EBNA-3, LMP-1, and LMP-2 have been described. EBNA-1 is responsible, among others, for suppressing the TP53 gene encoding the p53 protein, involved in the regulation of many cellular processes, such as DNA repair or the induction of apoptosis in response to DNA damage; EBNA-1 plays a major role in replication, activating transcription. EBNA-2, EBNA-3, LMP-1, and LMP2 are necessary for lymphocyte immortalization and their retention in the lymphoblastoid phase. LMP-1 disturbs cellular metabolism, especially regulation of apoptosis and cell cycle; it leads to an increase in cytokine production, increased expression of adhesion molecules, and anti-apoptotic genes $[54,55]$. The presence of EBV DNA is detected in $90 \%$ of gastric lymphoid epithelial carcinomas and in $5 \%-25 \%$ of gastric adenomas [14]. 
EBV and $H$. pylori cause chronic gastritis, and in the case of coinfection, the inflammatory process is significantly increased. H. pyloriand EBV infections alter miRNA expression in host cells, which modulates the host's inflammatory immune response, promoting bacterial survival in the gastric mucosa and inhibiting apoptosis in EBV positive cells [57].

\section{Proton pump inhibitors (PPI) and their influence on gastric microbiota}

PPIs have been among the most-prescribed drugs in the world since they were launched in the 1980s. They work by blocking the $\mathrm{H}+/ \mathrm{K}+$ ATPase of partial cells. They are used to treat peptic ulcer disease (PUD), $H$. pylori infection, dyspepsia, and gastroesophageal reflux disease (GERD). Regardless of other factors, PPIs can cause gastric atrophy. They inhibit the secretion of hydrochloric acid in the stomach, thus affecting the environment in the stomach causing hypergastrinemia and enterochromaffin cell hyperplasia [51, 58]. It is worth emphasizing that gastric acidity plays a key role in filtering out bacteria and preventing the development of enteric infection [19, 31]. Many published papers pay attention to the increase in gastric $\mathrm{pH}$ caused by the use of PPI, which can lead to bacterial overgrowth. In the presence of chronic hypochlorhydria the defense mechanism against pathogenic bacteria is weakened, which, among other things, results in infections caused by bacteria such as Clostridioides difficile and Campylobacter jejuni. Acid suppression leads to the growth of luminal and mucosal bacteria other than Helicobacter pylori, which can exacerbate chronic inflammation [16, 17]. The simultaneous occurrence of $H$. pylori and other bacteria has a synergistic effect on the induction of a higher level of serum cytokines and an increased risk of atrophic gastritis. Additionally, some studies indicate that bacterial migration from the antrum to the gastric body and fundus occurs with long-term use of PPI [58].

PPIs are used in the eradication of $H$. pylori. Eradication allows the reduction ofthe risk of gastric cancer and noncardia gastric cancer [59]. However, the beneficial effect of $H$. pylori eradication is limited to patients without baseline preneoplastic lesions [60, 61]. Eradication can reverse chronic gastritis in most and atrophic gastritis in some patients, whereas in the case of intestinal metaplasia it can only slow down the development of gastric cancer [18]. It is worth noting the growing $H$. pylori resistance to drugs. The increase in resistance to metronidazole, clarithromycin, and levofloxacin should be the reason for susceptibility testing before treatment [62].

Long-term use of PPI (over three years) and $H$. pylori infection act synergistically to increase the risk of gastric cancer [51]. Brusselaers et al. [63] also state that the risk of developing cancer increases with the duration of use of PPI: the higher risk is in the first three years, but after five years the risk decreases. According to Peng et al. [64], the use of PPI increases the risk of gastric cancer about 2.5 times among patients with GERD (gastroesophageal reflux disease). Additionally, GERD patients are more likely to develop cancers of the upper gastrointestinal tract [65].

During PPI therapy, the most numerous group of microorganisms are the Streptococcaceae, followed by the Prevotellaceae, Campylobacteraceae, and Leptotrichaceae [66]. In addition, patients on long-term PPI treatment have a greater abundance of Methylophilus compared with those on short-term PPI treatment [67]. Higher $\mathrm{pH}$ causes the growth of oral bacteria: Peptostreptococcus stomatis, Streptococcus anginosus, Parvimonas micra, Slackia exigua, and Dialister pneumosintes. In connection with the induction of various metabolic pathways, they may be involved in the progression of gastric cancer [42]. Also, the oral microbiota, which possesses nitrate reductase, produces $\mathrm{N}$-nitroso compounds from food nitrates, which are known gastric carcinogens [51].

Research is needed to determine the maximum duration of PPI use when they are therapeutically active and do not increase the risk of cancerogenesis. Data on PPI are divergent, although the fact that in the US, despite the widespread longterm use of $\mathrm{PPI}$, the occurrence of gastric cancer is relatively low is noteworthy [19]. There are also opinions like that of Zhang et al. [68] that PPIs can potentially be used as effective and relatively safe anticancer drugs that inhibit gastric cancer metastasis.

\section{Conclusions}

Biodiversity of microbiota of the human stomach may be more complex than we previously thought, and $H$. pylori colonization is that of the most important pathogen with clear impact on this diversity.

Despite the differences among various models and studies, there is strong evidence that the microbiota is necessary to maintain homeostasis and promote health, and that dysbiosis contributes to the host's susceptibility to infection and disease. The role of $H$. pylori in gastric microbiota is complex, and its connection with the inflammatory process and finally cancer has been proven. Another infectious agent, the Epstein-Barr virus, intensifies this process, which draws attention to the search for methods to identify the disease markers. Research in animal models could allow, in the future, the development of a strategy to manipulate the microbiota to prevent the development of stomach cancer. Also, with the help of molecular methods, which are increasingly used to explore the human microbiome, we can better understand the 
structure and relationships between individual microorganisms and then use this knowledge for diagnosis and therapy.

\section{Abbreviations}

CagA: cytotoxin-associated gene A, CFU: colony forming units, CMV: Cytomegalovirus, DNA: deoxyribonucleic acid, EBNA: Epstein-Barr nuclear antygen, EBV: EpsteinBarr virus, GERD: gastro-esophageal reflux disease, HERVs: human endogenous retroviruses, HHV6: human herpesvirus 6, IARC: The International Agency for Research on Cancer, IL-8: interleukin 8, LMP: latent membrane protein, LPS: lipopolysaccharide, miRNA: microRNA, rRNA: Ribosomalribonucleic acid, OTU: operational taxonomic units, PPI: proton pump inhibitors, PUD: peptic ulcer disease, T4SS: type IV secretion system, TCGA: The Cancer Genome Atlas, TGF- $\beta 1$ : transforming growth factor beta 1 , VacA: vacuolating toxin.

\section{Authors' Contributions}

M.D.: Drafting the article, literature review, writing the manuscript; D.P.-L.: Revising the article critically for important intellectual content, final proofreading and approval of the version for publication.

\section{Conflict of Interest}

The authors have no potential conflicts of interest to declare.

\section{References}

[1] NIH Human Microbiome Project http://www.hmpdacc.org (03.06.2020)

[2] Sender R., Fuchs S., Milo R.: Revised estimates for the number of human and bacteria cells in the body. PLoS Biol., 2016; 14: e1002533

[3] Rowan-Nash A.D., Korry B.J., Mylonakis E., Belenky P.: Crossdomain and viral interactions in the microbiome. Microbiol. Mol. Biol. Rev., 2019; 83: e00044-18

[4] Warren J.R., Marshall B.: Unidentified curved bacilli on gastric epithelium in active chronic gastritis. Lancet, 1983; 1: 1273-1275

[5] Hallen-Adams H.E., Suhr M.J.: Fungi in the healthy human gastrointestinal tract. Virulence, 2017; 8: 352-358

[6] Huseyin C.E., O'Toole P.W., Cotter P.D., Scanlan P.D.: Fortotten fungi - the gut mycobiome in human health and disease. FEMS Microbiol. Rev., 2017; 41: 479- 511
[7] Massarrat S., Saniee P., Siavoshi F., Mkhtari R., Mansour-Ghanaei F., Khalili-Samani S.: The effect of Helicobacter pylori infection, aging and consumption of proton pump inhibitor on fungal colonization in the stomach of dyspeptic patients. Front. Microbiol., 2016; 7: 801

[8] Wang Z.K., Yang Y.S., Stefka A.T., Sun G., Peng L.H.: Review article: Fungal microbiota and digestive diseases. Aliment. Pharmacol. Ther., 2014; 39: 751-766

[9] Khosravi Y., Gan H.M., Chia P.J., Croft L.J., Ng C.G., Goh K.L., Vellasamy K.M., Mariappan V., Ong K.C., Wong K.T., et al.: The gastric microbiome of four Malaysian gastroduodenal disease patients. Arch. Gene Genome Res., 2017; 1: 1-9

[10] Wołkowicz T., Januszkiewicz A., Szych J.: Gut microbiome and its dysbiosis as an important factor influencing the human health condition. Med. Dośw. Mikrobiol., 2014; 66: 223-235

[11] Huffnagle G.B., Noverr M.C.: The emerging world of the fungal microbiome. Trends Microbiol., 2013; 21: 334-341

[12] Karczewska E., Wojtas I., Trojanowska D., Budak A., ZwolinskaWcisło M., Wilk A.: Assessment of co-existence of Helicobacter pylori and Candida fungi in diseases of the upper gastrointestinal tract. J. Physiol. Pharmacol., 2009; 60: 33-39

[13] Lincoñir-Campos P., Merino-Barrera J.S., Smith C.T., GarciaCancino A.: Candida sp. as a potential reservoir and transmission facilitator of Helicobacter pylori. Biomed. J. Sci. \& Tech. Res., 2018; 4: 3736-3738

[14] Fol M., Jachowicz E.: Czynniki zakaźne w procesie nowotworzenia. Med. Og. Nauk. Zdr., 2016; 22: 7-14

[15] Armstrong H., Bording-Jorgensen M., Dijk S., Wine E.: The complex interplay between chronic inflammation, the microbiome and cancer: Understanding disease progression and what we can do to prevent it. Cancers, 2018; 10: 83

[16] Abbas M.K., Zaidi A.R.Z., Robert C.A., Thiha S., Malik B.H.: The safety of long-term daily usage of a proton pump inhibitor: A literature review. Cereus, 2019; 11: e5563

[17] Jump R.L.: Clostridium difficile infection in older adults. Aging Health, 2013; 9: 403-414

[18] Cheung K.S., Leung W.K.: Risk of gastric cancer development after eradication of Helicobacter pylori. World J. Gastrointest. Oncol., 2018; 10: 115-123

[19] Joo M.K., Park J.J., Chun H.J.: Proton pump inhibitor: The dual role in gastric cancer. World J. Gastroenterol., 2019; 25: 20582070.

[20] Global Burden of Disease Cancer Collaboration, Fitzmaurice C., Allen C., Barber R.M., Barregard L., Bhutta Z.A., Brenner H., Dicker D.J., Chimed-Orchir O., Dandona R., et al.: Global, regional, and national cancer incidence, mortality, years of life lost, years lived with disability, and disability-adjusted life-years for 32 cancer groups, 1990 to 2015: A systematic analysis for the global burden of disease study. JAMA Oncol., 2017; 3: 524-548

[21] Gunathilake M.N., Lee J., Choi I.J., Kim Y.I., Ahn Y., Park C., Kim $\mathrm{J} .:$ Association between the relative abundance of gastric micro- 
biota and the risk of gastric cancer: A case control study. Sci. Rep., 2019; 9: 13589

[22] National Cancer Institute, Surveillance Epidemiology, and End Results Program, https://seer.cancer.gov/statfacts/html/stomach. html (15.06.2020)

[23] The Cancer Genome Atlas Research Network: Comprehensive molecular characterization of gastric adenocarcinoma. Nature, 2014; 513: 202-209

[24] Seekatz A.M., Schinzlein M.K., Koenigsknecht M.J., Baker J.R., Hasler W.L., Bleske B.E., Young V.B., Sun D.: Spatial and temporal analysis of the stomach and smallintestinal microbiota in fasted healthy humans. mSphere, 2019; 4: e00126-19

[25] Nardone G., Compare D.: The human gastric microbiota: Is it time to rethink the pathogenesis of stomach diseases? United European Gastroenterol. J., 2015; 3: 255-260

[26] Sheh A., Fox J.G.: The role of the gastrointestinal microbiome in Helicobacter pylori pathogenesis. Gut Microbes, 2013; 4: 505-531

[27] Bassis C.M., Erb-Downward J.R., Dickson R.P., Freeman C.M., Schmidt T.M., Young V.B., Beck J.M., Curtis J.L., Huffnagle G.B.: Analysis of the upper respiratory tract microbiotas as the source of the lung and gastric microbiotas in healthy individuals. mBio, 2015; 6: e00037

[28] Malinowska M., Tokarz-Deptuła B., Deptuła W.: Mikrobiom człowieka. Post. Mikrobiol., 2017; 56: 33-42

[29] Rośniak-Bąk K., Pawłowska A., Paradowski M.: Helicobcter pylori: Cechy biochemiczne i patogenne bakterii oraz metody rozpoznawania zakażeń. Diagn. Lab., 2008; 44: 371-378

[30] Swora E., Stankowiak-Kulpa H., Marcinkowska E., Grzymisławski M.: Kliniczne aspekty diagnostyki zakażenia Helicobacter pylori. Now. Lek., 2009; 78: 228-230

[31] Minalyan A., Gabrielyan L., Scott D., Jacobs J., Pisegna J.R.: The gastric and intestinal microbiome: Role of proton pump inhibitors. Curr. Gastroenterol. Rep., 2017; 19: 42

[32] Brawner K.M., Kumar R., Serrano C.A., Ptacek T., Lefkowitz E., Morrow C.D., Zhi D., Kyanam-Kabir-Baig K.R., Smythies L.E., Harris P.R., Smith P.D.: Helicobacter pylori infection is associated with an altered gastric microbiota in children. Mucosal Immunol., 2017; 10: 1169-1177

[33] Noto J.M., Peek R.M.Jr.: The gastric microbiome, its interaction with Helicobacter pylori, and its potential role in the progression to stomach cancer. PLoS Pathog., 2017; 13: e1006573

[34] Ermolenko E., Varsin S., Baryshnikova N., Svarval A., Ferman R., Besedina N., Zakrevskaya A., Molostova A., Suvorov A.: Gastrointestinal dysbiosis accompanied of Helicobacter pylori infection and its correction by probiotic. J. Clin. Gastroenterol. Treat., 2018; 4: 055

[35] Eun C.S., Kim B.K., Han D.S., Kim S.Y., Kim K.M., Choi B.Y., Song K.S., Kim Y.S., Kim J.F.: Differences in gastric mucosal microbiota profiling in patients with chronic gastritis, intestinal metaplasia, and gastric cancer using pyrosequencing methods. Helicobacter, 2014; 19: 407-416
[36] Aviles-Jiminez F., Vazquez-Jiminez F., Medrano-Guzman R., Mantilla A., Torres J.: Stomach microbiota composition varies between patients with non-atrophic gastritis and patients with intestinal type of gastric cancer. Sci. Rep., 2014; 4: 4202

[37] Hu Y.L., Pang W., Huang Y., Zhang Y., Zhang C.J.: The gastric microbiome is perturbed in advanced gastric adenocarcinoma identified through shotgun metagenomics. Front. Cell. Infect. Microbiol., 2018; 8: 433

[38] Ferreira R.M., Pereira-Marques J., Pinto-Ribeiro I., Costa J.L., Carneiro F., Machado J.C., Figueiredo C.: Gastric microbial community profiling reveals a dysbiotic cancer-associated microbiota. Gut, 2018; 67: 226-236

[39] Castaño-Rodríguez N., Goh K.L., Fock K.M., Mitchell H.M., Kaakoush N.O.: Dysbiosis of the microbiome in gastric carcinogenesis. Sci. Rep., 2017; 7: 15957

[40] Yang I., Woltemate S., Piazuelo M.B., Bravo L.E., Yepez M.C., Romero-Gallo J., Delgado A.G., Wilson K.T., Peek R.M., Correa P., et al.: Different gastric microbiota compositions in two human populations with high and low gastric cancer risk in Colombia. Sci. Rep., 2016; 6: 18594

[41] Hsieh Y.Y., Tung S.Y., Pan H.Y., Yen C.W., Xu H.W., Lin Y.J., Deng Y.F., Hsu W.T., Wu C.S., Li C.: Increased abundance of Clostridium and Fusobacterium in gastric microbiota of patients with gastric cancer in Taiwan. Sci. Rep., 2018; 8: 158

[42] Coker O.O., Dai Z., Nie Y., Zhao G., Cao L., Nakatsu G., Wu W.K., Wong S.H., Chen Z., Sung J.J., Yu J.: Mucosal microbiome dysbiosis in gastric carcinogenesis. Gut, 2018; 67: 1024-1032

[43] de Leeuw M.A., Duval M.X.: The presence of periodontal pathogens in gastric cancer. Explor. Res. Hypothesis Med., 2020; 5: 87-96

[44] Salazar C.R., Francois F., Li Y., Corby P., Hays R., Leung C., Bedi S., Segers S., Queiroz E., Sun J., et al.: Association between oral health and gastric precancerous lesions. Carcinogenesis, 2012; 33: $399-403$

[45] Sun J.H., Li X.L., Yin J., Li Y.H., Hou B.X., Zhang Z.: A screening method for gastric cancer by oral microbiome detection. Oncol. Rep., 2018; 39: 2217-2224

[46] Wu J., Xu S., Xiang C., Cao Q., Li Q., Huang J., Shi L., Zhang J., Zhan Z.: Tongue coating microbiota community and risk effect on gastric cancer. J. Cancer, 2018; 9: 4039-4048

[47] Eusebi L.H., Zagari R.M., Bazzoli F.: Epidemiology of Helicobacter pylori infection. Helicobacter, 2014; 19: 1-5

[48] Kuklińska U., Łasica A.M., Jagusztyn-Krynicka E.K.: Białko CagA Helicobacter pylori - pierwsza zidentyfikowana bakteryjna onkoproteina. Post. Mikrobiol., 2011; 50: 97-106

[49] Mégraud F., Bessède E., Varon C.: Helicobacter pylori infection and gastric carcinoma. Clin. Microbiol. Infect., 2015; 21: 984-990

[50] Meng C., Bai C., Brown T.D., Hood L.E., Tian Q.: Human gut microbiota and gastrointestinal cancer. Genomics Proteomics Bioinformatics, 2018; 16: 33-49 
[51] Cheung K.S., Leung W.K.: Long-term use of proton-pump inhibitors and risk of gastric cancer: A review of the current evidence. Therap. Adv. Gastroenterol., 2019; 12: 1756284819834511

[52] Correa P.: Human gastric carcinogenesis: A multistep and multifactorial process - First American Cancer Society Award Lecture on Cancer Epidemiology and Prevention. Cancer Res., 1992; 52: 6735-6740

[53] Zhao Y., Zhang J., Cheng A.S., Yu J., To K.F., Kang W.: Gastric cancer: Genome damaged by bugs. Oncogene, 2020; 39: 34273442

[54] Bień S.: Rola infekcji wirusem Epsteina i Barr w schorzeniach głowy i szyi. Pol. Przegl. Otorynolaryngol., 2013; 2: 127-136

[55] Bocian J., Januszkiewicz-Lewandowska D.: Zakażenia EBV cykl życiowy, metody diagnostyki, chorobotwórczość. Postępy Hig. Med. Dośw., 2011; 65: 286-298

[56] Żuk-Wasek A.: Charakterystyka białek wirusa Epsteina-Barr - ich udział w zakażeniu latentnym i powiązanie z procesami nowotworzenia. Post. Mikrobiol., 2012; 51: 191-201

[57] Polakovicova I., Jerez S., Wichmann I.A., Sandoval-Bórquez A., Carrasco-Véliz N., Corvalán A.H.: Role of microRNAs and exosomes in Helicobacter pylori and Epstein-Barr virus associated gastric cancers. Front. Microbiol., 2018; 9: 63

[58] Bruno G., Zaccari P., Rocco G., Scalese G., Panetta C., Porowska B., Pontone S., Severi C.: Proton pump inhibitors and dysbiosis: Current knowledge and aspects to be clarified. World J. Gastroenterol., 2019; 25: 2706-2719

[59] Doorakkers E., Lagergren J., Engstrand L., Brusselaers N.: Helicobacter pylori eradication treatment and the risk of gastric adenocarcinoma in a Western population. Gut, 2018; 67: 2092-2096

[60] Arora N., Majumer K., Gupta A.: Gastric cancer and the microbiome. Asian Pac. J. Trop. Dis., 2015; 5: 353-35

[61] Wong B.C., Lam S.K., Wong W.M., Chen J.S., Zheng T.T., Feng R.E., Lai K.C., Hu W.H., Yuen S.T., Leung S.Y, et al.: Helicobacter pylori eradication to prevent gastric cancerin a high-risk region of China: A randomized controlled trial. JAMA, 2004, 291: 187-194

[62] Stauning A.T., Nordestgaard R.L.M., Frandsen T.H., Andersen L.P.: Gastric microbiota and resistance to antibiotics. In: Helicobacter Pylori - New Approaches of an Old Human Microorganism, eds.: B.M. Roesler. IntechOpen, London 2018

[63] Brusselaers N., Wahlin K., Engstrand L., Lagergren J.: Maintenance therapy with proton pump inhibitors and risk of gastric cancer: A nationwide population-based cohort study in Sweden. BMJ Open, 2017; 7: e017739

[64] Peng Y.C., Huang L.R., Lin C.L., Hsu W.Y., Chang C.S., Yeh H.Z., Kao C.H.: Association between proton pump inhibitors use and risk of gastric cancer in patients with GERD. Gut, 2019; 68: 374376

[65] Kim J.J.: Upper gastrointestinal cancer and reflux disease. J. Gastric Cancer, 2013; 13: 79-85

[66] Parsons B.N., ljaz U.Z., D’Amore R., Burkitt M.D., Eccles R., Lenzi L., Duckworth C.A., Moore A.R., Tiszlavicz L., Varro A., et al.: Comparison of the human gastric microbiota in hypochlorhydric states arising as a result of Helicobacter pylori-induced atrophic gastritis, autoimmune atrophic gastritis and proton pump inhibitor use. PLoS Pathog., 2017; 13: e1006653

[67] Shi Y.C., Cai S.T., Tian Y.P., Zhao H.J., Zhang Y.B., Chen J., Ren R.R., Luo X., Peng L.H., Sun G., Yang Y.S:. Effects of proton pump inhibitors on the gastrointestinal microbiota in gastroesophageal reflux disease. Genomics Proteomics Bioinformatics, 2019; 17: 52-63

[68] Zhang B., Ling T., Zhaxi P., Cao Y., Qian L., Zhao D., Kang W., Zhang W., Wang L., Xu G., Zou X.: Proton pump inhibitor pantoprazole inhibits gastric cancer metastasis via suppression of telomerase reverse transcriptase gene expression. Cancer Lett., 2019; 452: 23-30 